\title{
141. The Diuretic Action of Caffeine and Other Purine Derivatives in Japanese Toads.
}

\author{
By Toshiro Nishina. \\ (Pharmacological Laboratory, Tokio Imperial University.) \\ (Rec. July 24, 1926. Comm. by K. MrurA, M.I.A., Oct. 12, 1926.)
}

In commection with the investigations reported by Tamura and his co-workers, ${ }^{1)}$ it is advisable to examine the diuretic action of caffeine and its allies in the Japanese toad, which is very convenient for exact observations in applying various methods.

Caffeine diuresis in Japanese toads:

As in frogs, the diuretic action of the purine derivatives was found to be very slight in toads, and even when a most favorable dose for diuresis of caffeine, diuretin, agurin and theocin, was administered each being injected intravenously into urethanized toads in the quantity of 0.5 c.c. of $1 \%$ solution, the amount of urine excreted increased onls twice to that before the injection. Unlike in the mammals and thus in man, in toads the diuresis caused by theophylline and caffeine is more marked than that caused by theobromine.

Next, the renal portal vein and its branches in one of the kidneys was ligated, and observing the diuretic action of these drugs, it was found that the diuresis was induced in this operated kidney just as in the other normal one.

Caffeine diuresis in the isolated toad's kidney:

Further experiments were carried out on the excised kidney, perfused with the saline solution improved by Barkan, Bremser and Hahn ${ }^{2}$ under a constant pressure head of $25 \mathrm{~cm}$. of water in the aorta and $12 \mathrm{~cm}$. of water in the portal vein.

In order to determine the existence of a direct influence of purine derivatives on the glomerulus, all the renal portal veins in the isolated kidney were ligated, in accordance with Schmidt's method, ${ }^{3}$ leaving.

1) Tamura, Wataname, Shat, Komatsubara and Kimara. Proc. Imp. Academy, 2 (1926).

2) B.rkan, Bremser u. Hahn. Zeitschr. f. Biol., 74 (1921).

3) Schurot. Pflüger's Arch. f. d. ges. Physiol. 49 (1891), 34. 
No. 8] The Diuretic Action of Caffeine and Other Purine Derivatives in Japanese Toads. 443: only the passages in the renal arteries through which the perfusion fluid of 'various concentrations of purine derivatives (caffeine sodium benzoate: $1: 75.000-1: 200.000$, agurin, diuretin and theocin: each $1: 100.000-1: 200.000$ ) was poured into the glomerulus, and the rate of perfusion was measured. The results obtained shows, although the quantity of the perfusion fluid flowing through the kidney is always decreasing, indicating the induced vaso-constriction, the excretion of urine itself is clearly increasing. Thus the diuresis is caused by the direct renal action of those substances without a dilatation of the renal vessels.

To decide whether there is any direct influence on the tubules, the glomerulus and the tubules were perfused separately, (employing Bainbridge's method $\left.{ }^{1}\right)$, adding some of the purine derivatives in various. concentrations (1:200.000-1:300.000) only to the perfusion fluid to be poured into the tubules alone. Only a small fall of the perfusion rate and no change of the urine excretion indicate that there is no direct influence on the tubules to cause a diuresis.

To sum up the results of the preceding experiments on the isolated kidney, although the purine derivatives induce diuresis acting directly on the glomerulus, they do not exhibit any direct diuretic action on the tubules.

Caffeine diuresis caused by its extra-renal action :

With the method devised by the author (Fig. I.), the saline solution was poured directly into the glomerulus, and for the perfusion fluid to the tubules the author used the one to which the so-called extra-renal factor of purine derivatives had been added by perfusing a hind leg. In the case of all the purine derivatives used (each in concentration of $1: 200.000$ ), a noticable increase in the urine excretion was caused, this being the evidence that the purine derivatives have a diuretic influence indirectly on the tubules.

From these experiments, the author has come to the conclusion that the mode of diuretic actions of the purine derivatives, can be divided at least into two, i. e., the direct action on the glomerulus and the indirect action on the tubules.

1) Bainbridgr, Menzies and Collins. Journ. Physiol., 48 (1914), 233. 
Fig. I.

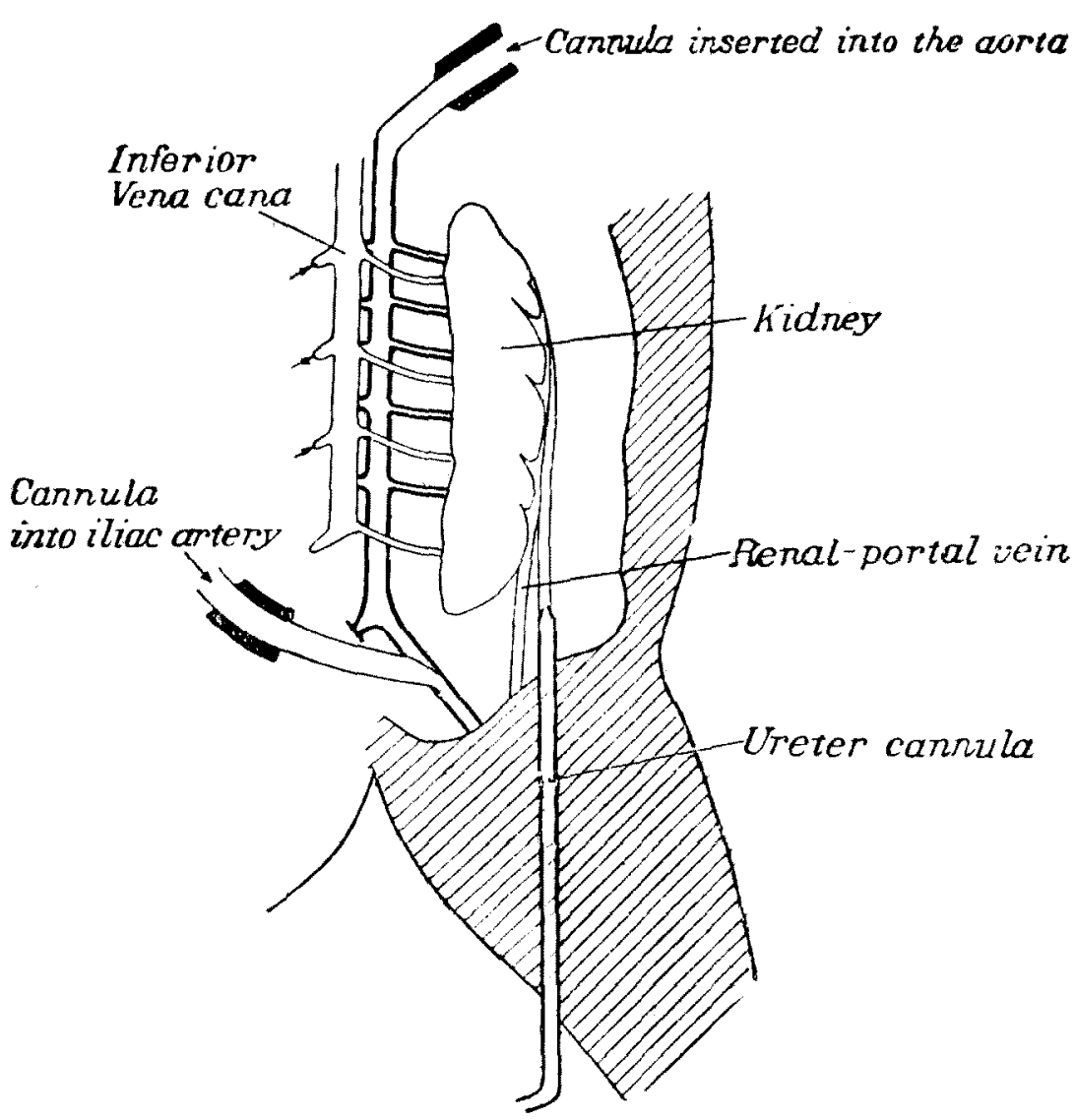

Fig. I. A new periusion method of investigation of the extra-renal action of drugs on the tubules. 